\title{
Discovery of a sol-gel reinforcing the strength of hair structure: mechanisms of action and macroscopic effects on the hair
}

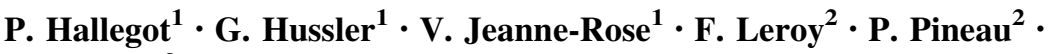 \\ H. Samain ${ }^{3}$
}

Received: 29 September 2015/ Accepted: 6 January 2016/Published online: 5 February 2016

(C) The Author(s) 2016. This article is published with open access at Springerlink.com

\begin{abstract}
The human hair presents a fibrillar structure, composed of keratinized cells, covered by a wall of cellular scales, piled up like tiles on a roof. Substantial variations in diameter exist in the population. Fine hair is a major concern for women and men. Hair may lack volume and lack of firmness and bounce. Hairstyles lack volume and hold, and hair is flat and difficult to style. To strengthen the hair structure, penetration of materials, like polymers, could be a realistic approach. The hair cuticle barrier, however, prevents big molecules to penetrate. To overcome this hurdle, reactive sol-gel have been studied, leading to the identification of the 3-aminopropyltriethoxysilane. Smaller than polymers, this silane has been studied on the key question of the penetration. After having understood its potential in penetration, the effect on mechanical transformation has been studied. The objective of this work was also to evaluate the efficacy of the 3-aminopropyltriethoxysilane, formulated in products for fine hair to respond to the needs of both feminine or masculine head hair. Several studies and techniques were
\end{abstract}

used to determine the impact of the optimal efficacy of 3-aminopropyltriethoxysilane by provisory transforming hair fiber.

Graphical Abstract To improve hair resistance, reactive sol-gels have been studied, leading to the identification of the 3-aminopropyltriethoxysilane. After treatment, material is detected inside the fiber. The resistance of material to shampoos leads to think that the molecules reacted to oligomers. Several studies and techniques were used to determine the impact of the optimal efficacy of 3-aminopropyltriethoxysilane. The three-point bending measurements showed that 3-aminopropyltriethoxysilane improves the mechanical resistance of dry hair. In addition, the morphology of the surface revealed an absence of a perceptible coating. These results explain the effect of body given to fine hair, and its remarkable natural touch. Other experiments have been done to elucidate the intimate effect of material on the complex structure of hair.
H. Samain

HSAMAIN@rd.loreal.com

L'Oréal Research and Innovation, Aulnay-sous-bois, France

L'Oréal Research and Innovation, Asnières-sur Seine, France

3 LOréal Research and Innovation, Chevilly-Larue, France 


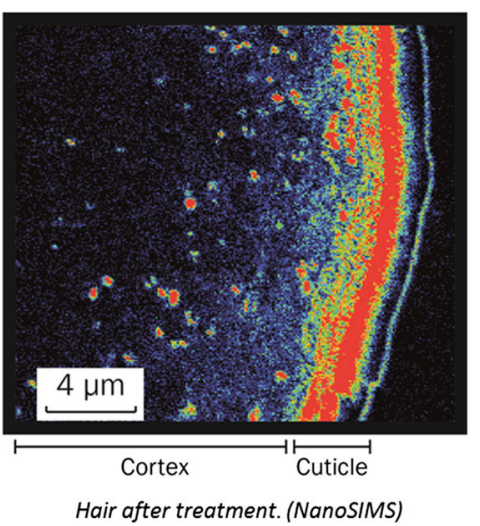

Keywords 3-aminopropyltriethoxysilane - Mechanical reinforcement · Penetration · Fiber · Hair · Fine hair . Bending

\section{The context and the objective}

The human hair presents a fibrillar structure composed of keratinized cells covered by a wall of cellular scales piledup like tiles on a roof [1]. The diameter of adult hair ranges between 40 and $120 \mu \mathrm{m}$. This substantial variation is related to several factors among which are the size of dermal papilla, ethnic origin and age [2-6]. Fine hair is a major concern for women and men.

Beyond the diameter, self-perception of fine hair is strongly linked to the mechanical properties of fibers when one touches and manipulates his/her own hair. Hair may lack firmness and can be difficult to style. Head of hair lacks volume, bounce, and hold. In the cosmetic field, fine hair is considered as a frequent concern that deserves special attention and customized solutions.

To give more firmness to fine hair, solutions exist, like the so-called styling products [7], which apply a solubilized polymer that forms, after drying, a coating of material on the surface of each fiber. The coating is very thin (usually less than one micrometer), but can confer a self-perceived effect of "body." Mechanical measurements show that the effect is more due to increased interactions between fibers rather than an individual increase of strength of each hair [8]. Such an approach does not, however, last over 1 or 2 days since in real life, and the surface of hair is subject to strong hydric and mechanical stresses (washing, combing, and frictions).

To improve fine hair condition with long-lasting efficacy, penetration of a material inside the fiber appears as a logical approach to being considered. Indeed, mechanical and hydric stresses are none or less important in the fiber than on the surface. Hence, we can hypothesize that penetrated material can last much more than the material on the surface. As a proof of this hypothesis, the oxidative coloring, the main hair dyeing technology, works this way: Dye molecules penetrate the hair and remain for a rather long time, usually several weeks.

If penetration of material into the fiber appears as a potential solution to give mechanical improvement to fine hair, the question then arises to which material could be used for that purpose.

Large polymers or particles can provide hard material, but cannot penetrate into hair. The scales of the hair and the tight structure of the keratins do not allow any large molecules to penetrate [9]. Therefore, it is logical to abandon the idea of using large molecules and, rather, to focus on smaller-sized molecules.

To solve the problem, the conceptual idea to create material, in situ, has been studied. For that, sol-gel strategy has been chosen because of several potential advantages. Sol-gels can react and give hard material, and the molecules can be small in size and soluble in water, i.e., two main points favorable for hair penetration. In addition, the reaction conditions are mild and compatible with safety and comfort conditions required for personal usage. To conduct this work, we began in an exploratory approach. After identification of penetrating molecules, we studied the effects of the most interesting molecules on hair behavior.

\section{Identification of the sol-gel system}

Sol-gel systems undergo two successive reactions: hydrolysis and condensation. The kinetics of condensation is a key point since, in our concept, the molecules need to penetrate before reaching a too high condensation level. Thus, we focused on sol-gel systems the reactivity of 
which can be classed as "intermediate." This "intermediate" reactivity means that the kinetics of condensation is rapid, but less than the kinetics of penetration.

Taking into consideration that penetration usually takes place within 5-20 min (classical time for a hair treatment), this led us to eliminate very rapidly reacting sol-gels. Silanes were selected due to their large range of kinetics of reaction (from very slow to rapid) and the possibility to use waterborne compounds $[10,11]$ (water being the commonest companion of the hair cosmetic field).

To study the silane penetration and select the most interesting systems, several silanes were tested, in adapted solvents (water or ethanol) and different concentrations (from 1 to $15 \%$ ). Each solution was applied in a rinsed procedure (which differs from styling product applied in a leave-on procedure). The rinsed procedure presents the advantage of removing material from the surface. Therefore, by washing out the material at the surface, the silicon analyses of the hair give unambiguous conclusions on the amount y of material that has penetrated.

A typical procedure implies the application of the solution on hair swatches. After 10-15 min, at room temperature, the hair is rinsed, washed, and analyzed. We used three techniques:

1. X-ray fluorescence (XRF) for the global analysis of silicon atom. It does give a first answer on the total amount of silicon element that stays after treatment. It also can be used to follow the lasting over shampoos.

2. Scanning electron microscopy with X-ray analysis to visualize silicon into the hair at micron scale.

3. Secondary ion mass spectroscopy (NanoSIMS) to determine the location of silicon within the hair fiber

Several tetra-, tri-, or bi-alcoxysilane have been studied: Tetraethoxysilane (TEOS), methyltriethoxysilane, 3-aminopropylmethyldiethoxysilane, 3-aminopropyltriethoxysilane, 3(-2-aminoethylamino)propyltrimethoxysilane, N-méthylaminopropyltrimethoxysilane, 3-2-2-aminoethylaminoethylaminopropyltrimethoxysilane, dimethyl diethoxysilane (DMDES), and 3-(tri(ethoxy/methoxy)silylpropyl)urea were supplied by Sigma-Aldrich (3050 Spruce St. St. Louis, MO 63103, USA), and 4-aminobutyltriethoxysilane and N-6aminohexylamino-propyltrimethoxysilane were supplied by Gelest (11 East Steel Road, Morrisville, PA 19067, USA)

Hair penetration is generally rather low and can be below the limit detection of XRF, like in the case of TEOS and DMDES, even when tested at $15 \%$.

The most performing molecules, in terms of penetration and uptake, were gamma-amino propylsilanes. In particular, 3-aminopropyltrimethoxysilane was found as one of the most interesting [12].

\section{Penetration and uptake of material}

The penetration of this silane material within the hair structure after one application is moderately deep. The precise analysis confirmed this material has penetrated and stands below the surface, in the hair peripheral cortex (Fig. 1).

The question of the state of the material in the fiber was also studied. Due to analytical limits, we were not able to have a direct answer on the condensation state of the material, but several observations led us to think that a substantial part of the molecules has condensed into polymers:

1. Extraction of hair with water: This extraction consists in leaving the hair under stirring for an optimized time in water and to analyze the residue present in water. Silane compounds were found among the extract mixture. After this extraction, the hair continued showing an important remaining content of silicon. To explain this long lastingness, we can postulate that the formed material is linked to chemical functions of the hair material (i.e., ionic interactions), or is trapped due to its molecular size. Since the oligomers are cationic and also can chemically react to condensate into large-sized molecules, both explanations coexist.

2. In real life, hair is highly solicited by shampoos. The analyses of the hair, several weeks and sometimes months after the treatment, show that silicon remains in relative important amount. We wondered whether the uptake could be limited by the ability of the hair structure to capture the molecules. Hence, we performed tests with several applications and continued observing a high level of persistence of silicon (Fig. 2).

Hair (bleached) has been treated three times by the following procedure: application of $10 \% 3$-aminopropyltrimethoxysilane in water followed by rinsing $15 \mathrm{~min}$ later. Silicon amount measurements (XRF) were performed after the treatments, and after 3, 5 and 10 shampoos. Amounts are expressed from silicon as PDMS (polydimethylsiloxane) equivalent.

\section{Measurement of the mechanical impact}

The mechanical impact of this treatment on the hair was addressed by measuring the resistance to bending. As shown in Fig. 3, the bending resistance is evaluated by a three-point stimulation of a lock of hair. The resistance measurement gives an interesting response as a direct proof of effect. A second interest of this technique is that the generated movement mimics, to a certain extent, those of 
Fig. 1 Solution of $10 \%$ 3-aminopropyltrimethoxysilane in water has been prepared. $\mathrm{pH}$ has been decreased to $\mathrm{pH} 9$ by adding lactic acid. The solution was applied onto hair. Rinsing was performed $10 \mathrm{~min}$ later. Analysis was carried out through NanoSIMS

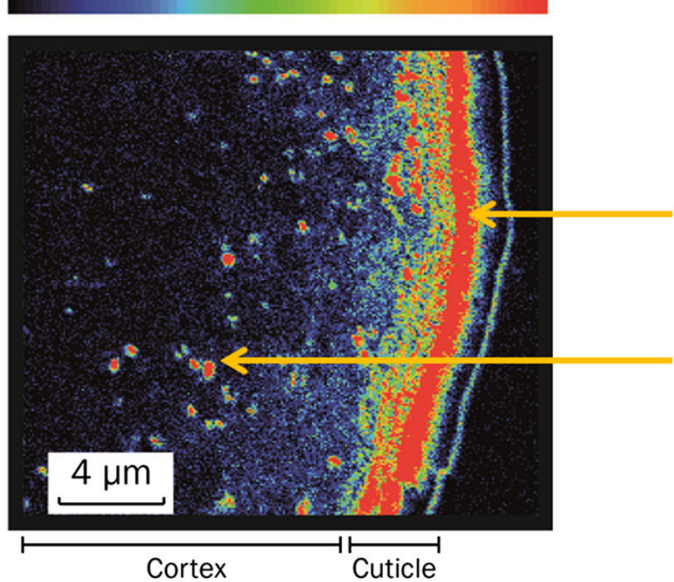

Silicon in the hair cuticle and in the peripherial cortex

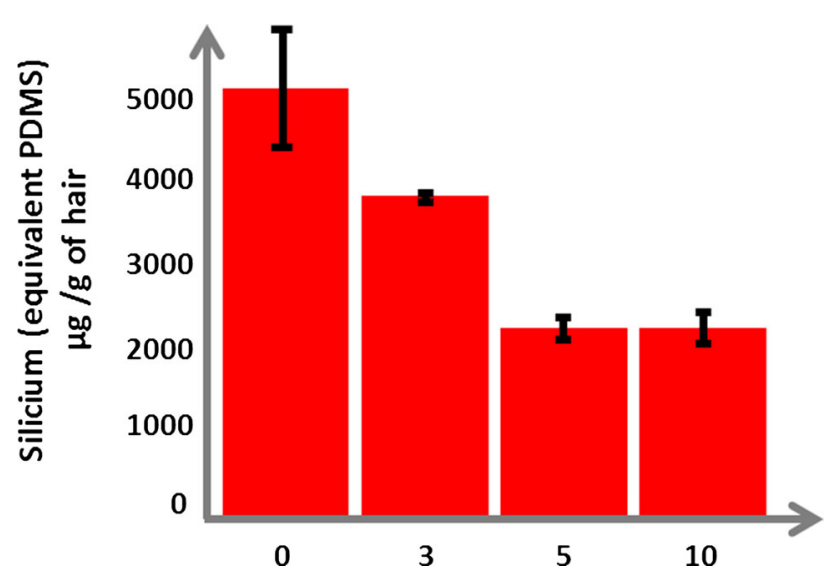

Fig. 2 Remaining silicon in hair after 0, 3, 5, 10 shampoos

people in the real life when touching and/or styling their hair.

The treatment led to a substantial increase in resistance to bending on dry hair. For instance, a treatment with 3 -aminopropyltrimethoxysilane $10 \% \mathrm{pH} 9$ in water for 15 min followed by rinsing and drying led to an increase in the bending force by $17 \%$ as compared to untreated. The same procedure applied 10 successive times enhanced the resistance to bending by $24 \%$. More maximized conditions can even enhance more the resistance to bending. For instance, a treatment with 3-aminopropyltrimethoxysilane $10 \%, \mathrm{pH} 10$ in water for $30 \mathrm{~min}$ at $40{ }^{\circ} \mathrm{C}$ increased the resistance to bending by $46 \%$. To our knowledge, such improved bending resistance has never been observed with any other marketed hair care product.

The same evaluation was carried out on wet hair and showed no substantial effect on the bending resistance. The effect appears then as selective (increase in resistance to bending on dry hair but not on wet hair), which is very appreciable when considering the needs of people with fine hair. These people look for an increase in "body" for their dry hair, without changing that of wet hair.

In addition, treated hairs were evaluated by both panel experts and subjects: Hair offered a natural touch and remarkably different from the one felt after application of styling products. The morphology of the surface (Fig. 4) revealed an absence of a perceptible coating.

Such absence of surface coating indicates that the increase in resistance to bending was not linked to material

Fig. 3 Three-point bending test (length of hair: $37 \mathrm{~mm}$, Quantity of hair; $20 \mathrm{mg}$. Penetration of the probe: $100 \mathrm{~mm} \mathrm{~min}{ }^{-1}$. Depth of penetration: $5 \mathrm{~mm}$ )

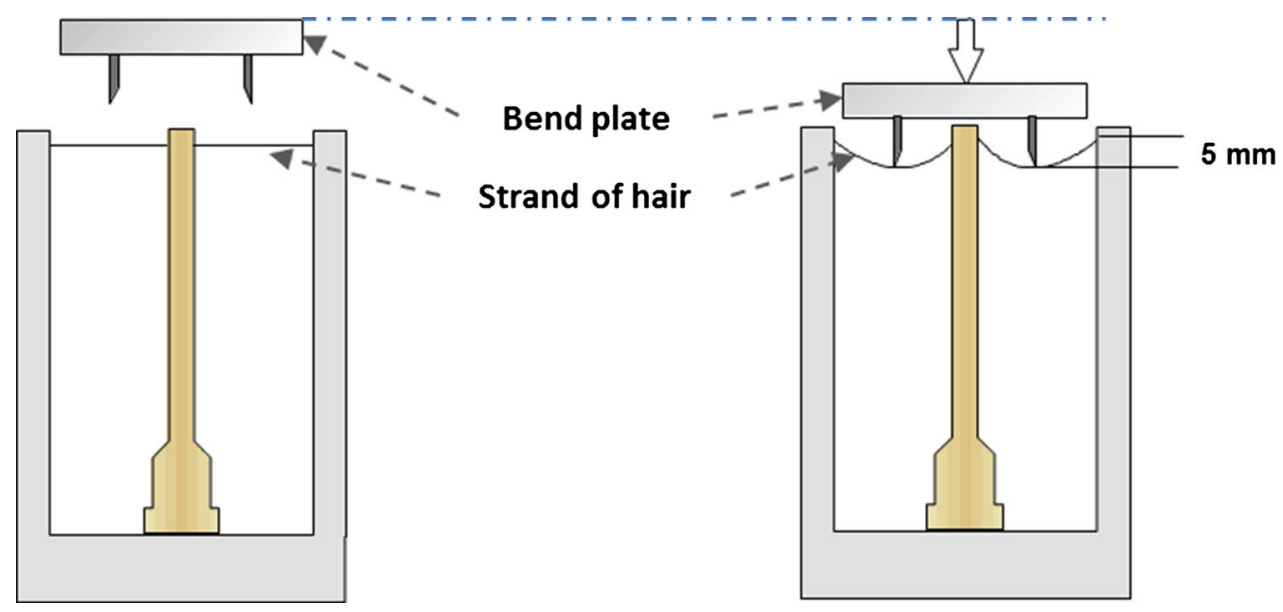



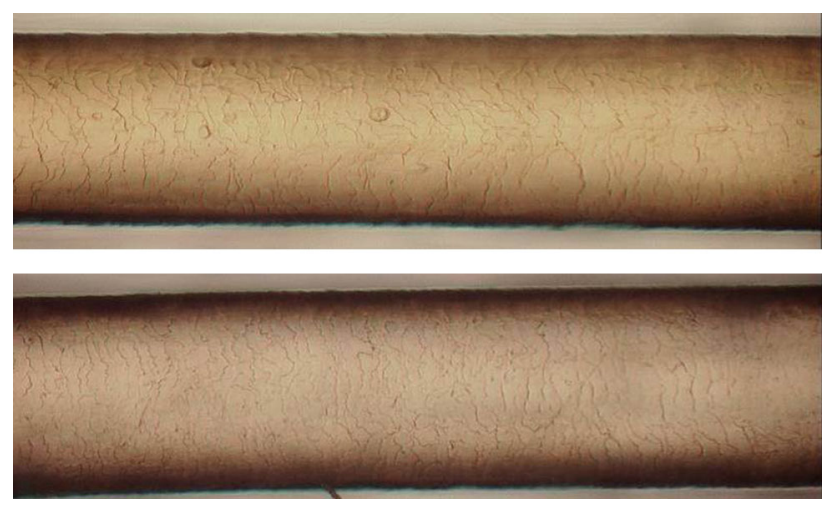

Fig. 4 Optical microscopy with confocal reconstruction. Hirox KH7700 (Hirox Co., Ltd-Japan). Up Untreated, Down Treated by a 3-aminopropyltrimethoxysilane solution in water, rinsed after $15 \mathrm{~min}$

deposit on the surface, but rather to the presence of the silane material within the fiber.

\section{Possible scenario of the different steps of transformation of the tested silane derivative}

1. Chemical analyses show that the ethoxy groups are quickly hydrolyzed when 3-aminopropyltrimethoxysilane monomer is being mixed with water. The silanol monomer is also rapidly transformed into its dimer, yielding condensation products of molecular weight below $1500 \mathrm{Da}$ [13].

2. During the application step, molecules can penetrate. As a matter of evidence, the fact that some material remains within the fiber after rinsing proves that the molecules are of a size small enough to substantially cross the hair cuticle.

3. To understand why and how the oligomers that have penetrated are not washed out during the rinsing step, we hypothesized that their amino groups interact with the numerous internal anionic groups of hair (carboxylic or sulfonic groups).

4. During drying, we assume that the oligomers undergo a condensation process that cannot be demonstrated by direct measurement. The fact that the material can be found within the hair fiber after several weeks or months (corresponding in some cases to 40 to 60 shampoos and some thousands of liters of water) fits with this assumption. However, this exceptional lasting does not prove by itself that the silane molecules condensate within the hair matrix. Other hypotheses, like binding to proteins [14-16], could explain the phenomenon as well.

5. The clear appearance of the hair surface after rinsing (devoid of any coating) can also be explained when considering the water solubility of the oligomers. Finally, this phenomenon can be seen as an interesting selective process: Oligomers that have penetrated the fiber are trapped and remain within it, whereas those present at the surface are eliminated during rinsing. Thanks to this selective behavior, the hair is given an increased resistance to bending and presents a touch of natural hair.

\section{Discussion on the origin of the bending reinforcement}

An interesting question that arises is the intimate origin of the observed mechanical effect.

The content of silane material within the fiber (calculated by XRF) was found below $1 \%$ versus hair weight), i.e., a rather small figure in comparison to the strong mechanical effect brought to the bending resistance.

- The silicon-based material formed into the hair may be hard, albeit of a lower hardness than the hair material itself (Young modulus around $4 \mathrm{GPa}$ ).

- The location of the material within the fiber might be a key point. As described above, the silicon is found in the peripheral region of the hair cortex. It has been shown that a material present at the edge of the hair is more effective in reinforcing resistance to bending than a material evenly spread in the diameter. [17]

These two considerations apart, a third important phenomenon has to be taken into account.

$\mathrm{X}$-ray microdiffraction analysis of the hair before and after treatment revealed an increase in the distance between fibrils of keratin. Usually of 90 Angströms [18], this distance raised up to 96 Angströms (in average). Theoretical calculations indicate that such an increase in distance corresponds to a $28 \%$ increase in surface of the keratinous associated proteins (KAP) forming the cement between the fibrils, as described in the Fig. 5.

To our knowledge, it is the first time that such phenomenon is reported on dry hair, suggesting that the oligomers have partially filled the amorphous keratin material and separated the fibrils from each other. Other measurements should obviously be carried out to confirm this possible scenario. It is nevertheless reasonable to view the tested silane compound as an internal hair expander.

The intimate interactions, at the molecular level, between the oligomers and hair proteins remain a key aspect to being further explored. In particular, are these ensured by ionic interactions and/or covalent bonds? The complexity of the hair structure leaves place to complex scenarios of inter-molecular re-arrangements in which, for 


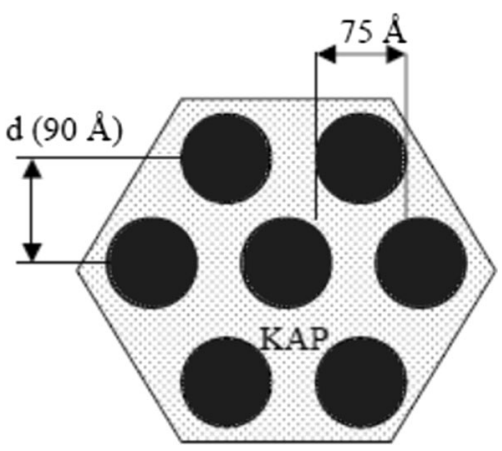

Fig. 5 Keratin fibrils (in black) are separated by amorphous proteinic cement (in gray). The increase in interfibrillar distance (90 to 96 Angströms in average) causes an increase in the surface of proteinic cement by $28 \%$

instance, the silanols and the amino group could be involved [19].

\section{Conclusions}

A new approach in the hair care cosmetic field is proposed for fulfilling the specific needs of fine hair.

- The chemistry, "mild \& reactive," complies with a cosmetic usage.

- The approach can be seen as a "spare part solution": The material is brought to the hair as small molecules. These can penetrate the hair and further add substance within its structure. The uptake of the material by hair is undeniable, and the increased resistance to bending remaining over time leads to hypothesize that molecules are trapped and condensed.

Applied to hair, this practical approach gives a useful and appropriate "body effect" that makes the hair felt stronger than originally. This effect is paramount to people that self-perceive their hair as fine, and more especially to those complaining about hair lacking firmness and bounce

Elucidating the intimate interactions of the material with the keratin microstructure remains an important objective of future studies.

Acknowledgments We warmly thank Professor Clément Sanchez (Department of Hybrid Material Chemistry, Collège de France, Paris, France) for the exploratory phase, and Jean Doucet and Novitom company for the X-ray study and interpretation.

Open Access This article is distributed under the terms of the Creative Commons Attribution 4.0 International License (http://crea tivecommons.org/licenses/by/4.0/), which permits unrestricted use, distribution, and reproduction in any medium, provided you give appropriate credit to the original author(s) and the source, provide a link to the Creative Commons license, and indicate if changes were made.

\section{References}

1. Muto H, Ozeki N, Yoshioka I (1981) Fine structure of the fully keratinized hair cuticle in the head hair of the human. Acta Anat (Basel) 109(1):13-18

2. Loussouarn G, El Rawadi C, Genain G (2005) Diversity of hair growth profiles. Int J Dermatol 44(Suppl 1):6-9

3. Baque CS, Zhou J, Gu W et al (2012) Relationship between hair growth rate and morphological parameters of human straight hair: a same law above ethnical origins? Int J Cosmet Sci 34(2):111-116

4. C Bouillon, J Wilkinson (eds) (2005) The science of hair care, 2nd edn. Taylor and Francis, London, p 2-29

5. Vernall DG (1961) A study of the size and shape of cross sections of hair from four races of men. Am J Phys Anthropol 19:345-350

6. Otsuka H, Nemoto T (1988) Study on Japanese hair. Koshokaishi 12:192-197

7. C Bouillon, J Wilkinson (eds) (2005) The science of hair care, 2nd edn. Taylor and Francis, London, p 169-179

8. J Gray, J Thomas (2010) Textbook of cosmetic dermatology, 4th edn. Informa Health Care, London, p 225-227

9. Morel O, Christie RM, Greaves A, Morgan KM (2008) Enhanced model for the diffusivity of a dye molecule into human hair fibre based on molecular modelling techniques. Coloration Technol 124(5):301-309

10. B Arkles, JR Steinmetz, J Zazyczny, P Mehta (1992) Silanes and other coupling agents. In: KL Mittal (ed), VSP, Utrecht, pp 91-104

11. Brochier Salon MC, Bayle PA, Abdelmouleh M, Boufi S, Belgacem MN (2008) Kinetics of hydrolysis and self condensation reactions of silanes by NMR spectroscopy. Colloids Surf A Physicochem Eng Asp 312(2-3):83-91

12. Samain H, Rollat I, Jeanne-Rose V, Sanchez C, EP 1216023 A1

13. Jeanne-Rose V (2013) Hair' S 13; 18th hair science symposium. Lubeck Germany, 4-6 Sept 2013

14. Sapsford KE, Ligler FS (2004) Biosens Bioelectron 19:1045-1055

15. El-Ghannam AR, Ducheyne P, Risbud M, Adams CS, Shapiro IM, Castner D, Golledge S, Composto RJ (2004) J Biomed Mater Res A 68:615-627

16. Balasundaram G, Sato M, Webster TJ (2006) Biomaterials 27:2798-2805

17. As known in general mechanic science, the bending for a given force is linked to the moment of inertia of the structure. The moment of inertia, in the case of a cylinder, is proportional to the square of the distance of the material to the axis. Hence, the peripheral distribution of hard material appears the best to impact the bending resistance

18. Kreplak L, Franbourg A, Briki F, Leroy F, Dallé D, Doucet J (2002) A new deformation model of hard a-keratin intermediate filament mechanical properties. Biophys J 28:2265-2274

19. Young JK, Nuriye K, Chang Hoon N (2012) IBC 4(9):1-7 